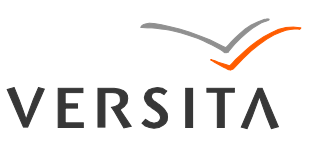

GEOCHRONOMETRIA 40(4) 2013: 220-228

DOI 10.2478/s13386-013-0110-0

Available online at

www.springerlink.com

\title{
LEXSYG - A NEW SYSTEM FOR LUMINESCENCE RESEARCH
}

\author{
DANIEL RICHTER ${ }^{1,2}$, ANDREAS RICHTER ${ }^{1}$ and KAY DORNICH ${ }^{1}$ \\ ${ }^{\text {I}}$ Freiberg Instruments GmbH, Delfterstr. 6, 09599 Freiberg, Germany \\ ${ }^{2}$ Geographisches Institut, LS Geomorphologie, University of Bayreuth, Universitätsstr. 30, 95447 Bayreuth, Germany
}

Received 30 January 2013

Accepted 21 March 2013

\begin{abstract}
The lexsyg luminescence system is the brainchild of the late Matthias Krbetschek, to whom this paper is dedicated by his colleagues and friends.
\end{abstract}

\begin{abstract}
The newly developed lexsyg system by Freiberg Instruments is a versatile luminescence reader, suited for research on the luminescence of materials, fundamental research in luminescence dating, but also for routine mass measurements in retrospective dosimetry as well as in dating application. The 80 sample storage wheel is disconnected from the measurement chamber and therefore crosstalk of optical stimulation is absent and cross-irradiation is negligible from the $\alpha$ - and $\beta$-sources, which are providing very uniform irradiations, with the latter especially designed for radiofluorescence (RF) measurement. Optical excitation sources and filter wheels to vary detection wavelengths can be programmed to change at almost any time within measurement sequences, including the automated change of an optional wheel holding up to four different detectors. Thermoluminescence measurements and preheating are possible with a versatile heater, which can be programmed for linear or non-linear heating or cooling, as well as holding a temperature constant. Rates as well as durations can be varied, together with individual ramping, staging and cooling for an almost unlimited number of steps. Violet- and IR-lasers, green and blue LED-arrays can be operated in continuous (CW) or modulated mode (linear/non-linear), and optionally for pulsed as well as time resolved luminescence detection. Six arrays of power LEDs allow the simulation of different bleaching regimes ('solar simulator'), while luminescence detection can be achieved by a variety of photomultiplier tubes and by CCD cameras for spatially resolved measurements and luminescence spectra.
\end{abstract}

Keywords: luminescence equipment, dosimetry, luminescence dating, luminescence detection.

\section{INTRODUCTION}

The detection of luminescence emissions is often used for dosimetric application and fundamental material research. It can be employed for retrospective dosimetry of environmental (e.g. as applied in dating) or accidental character. Various materials with different properties are

Corresponding author: D. Richter

e-mail: daniel.richter@freiberginstruments.com employed, which, in either case, requires fundamental research and subsequently routine mass measurements. Such a diversity of applications requires either very specific equipment, which is custom or in-house made, or versatile research instruments which can be used for both fundamental research and routine measurements.

Existing systems for luminescence research (e.g. Bortolot, 2000; Bortolot and Carriveau, 1982; BøtterJensen and Bundgaard, 1978; Lapp et al., 2012; Mejdahl, 1982) provide the means to measure luminescence with 
several excitation sources, but are usually limited in luminescence detection. Changing detectors or detection windows often requires manipulation of the equipment and thus the measurement of the same sample aliquot with different detectors or in different wavelength bands is neither feasible nor possible within a measurement sequence. This is potentially a limitation in research and the development of new approaches. More versatile and more flexible instruments are needed, which allow more diverse ways to perform luminescence stimulation and measurement without having to change parts of the equipment during or between measurement sequences.

Here we describe a newly developed luminescence measurement system - lexsyg - which can be used for many purposes in fundamental material and dosimetric research, as well as retrospective dosimetry and dating.

\section{LUMINESCENCE OF NON-CONDUCTING SOLIDS}

Luminescence of minerals and other phosphors is used in dosimetry or dosimetric dating as well as in fundamental material research. Such materials include natural and artificial minerals, but also artificial compounds and objects of our daily life. The fundamentals and basics of luminescence research have been summarized elsewhere, e.g. in Aitken (1985; 1998), Bøtter-Jensen et al. (2003), McKeever (1985), Yukihara and McKeever (2011). The aim of the lexsyg system is towards research in dosimetry, material properties and especially dosimetric dating. The requirements for these applications provide the basis for the luminescence system, but are not limited to these, and the versatility of the lexsyg system appears to be especially useful for fundamental research.
Fig. 1 provides an overview of the luminescence emissions of the two most frequently used natural minerals (quartz and feldspar) in luminescence dating as examples of the application of the lexsyg system. Information is provided on the two essential processes - stimulation and detection. Wavelengths for various luminescence emissions and excitation sources as well as detection ranges and sensitivities for detectors suited for these two minerals are shown (Fig. 1).

\section{THE LEXSYG SYSTEM}

lexsyg is a highly flexible modular system (Fig. 2) for the measurement of thermally or optically stimulated luminescence (i.e. TL or OSL/IRSL) and fluorescence excited by ionizing radiation. It is designed for maximised versatility to provide a wide range of stimulation and detection options for luminescence research. The modular design allows easy modification for almost any kind of user defined configuration of luminescence excitation and detection, optionally based on an automatic detector changer.

\section{General concept}

The general concept of the lexsyg follows the separation of sample aliquot storage from the aliquot measured, in order to eliminate cross bleaching effects during optical stimulation/bleaching and minimize cross-irradiation (Richter et al., 2012). This is achieved by storing the aliquots awaiting measurement on an 80 position wheel (cup/disc $\varnothing=10 \pm 0.1 \mathrm{~mm}$ ) in the storage chamber and a pick-and-place system for the aliquot to be measured, allowing independent operation of measurement chamber and sample-disc reservoir (Fig. 3).

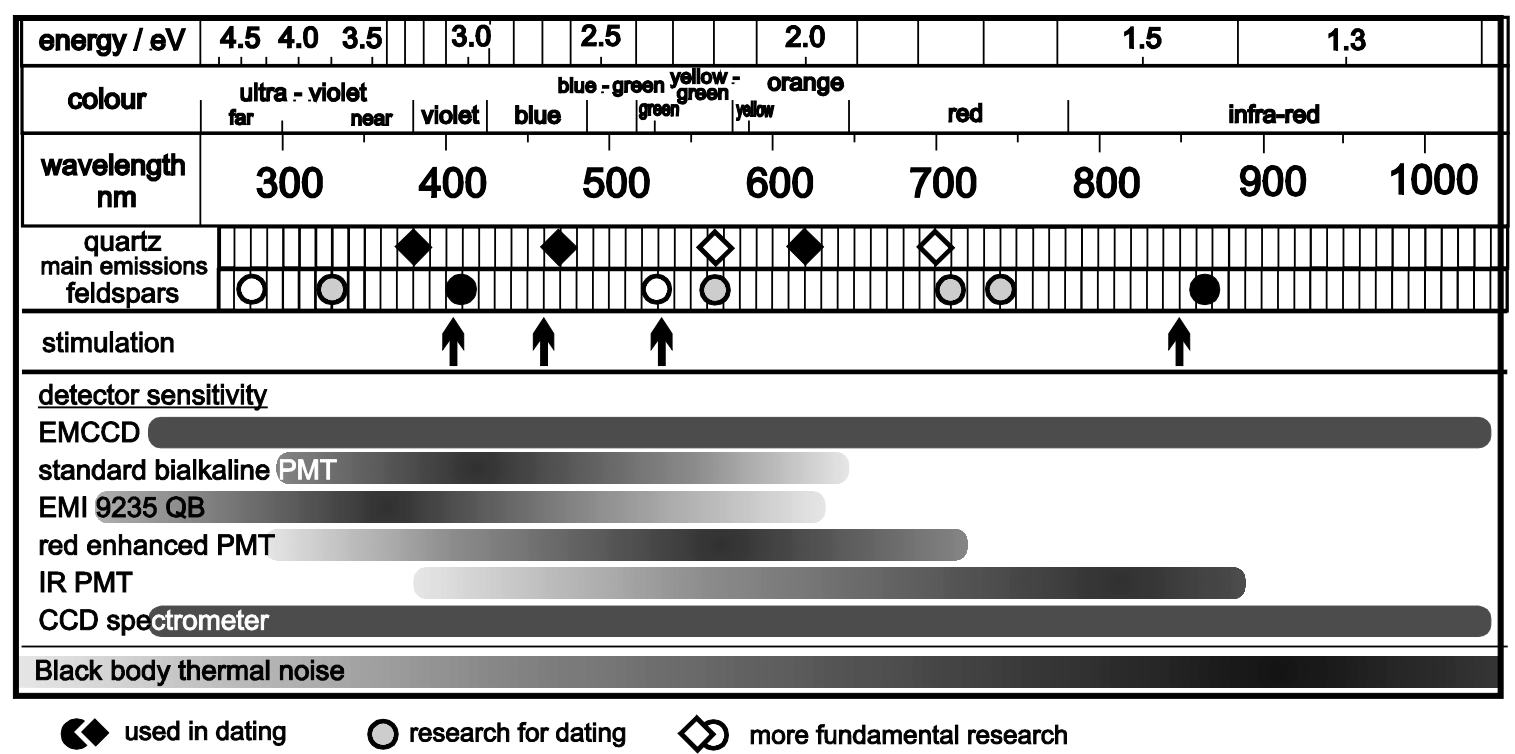

Fig. 1. Wavelengths of the main luminescence emissions of the natural minerals quartz (diamonds) and feldspar (circle) with stimulation wavelength bands of lasers and LEDs. The approximate sensitivity of various detectors is indicated by the intensity of the coloration over wavelength. 


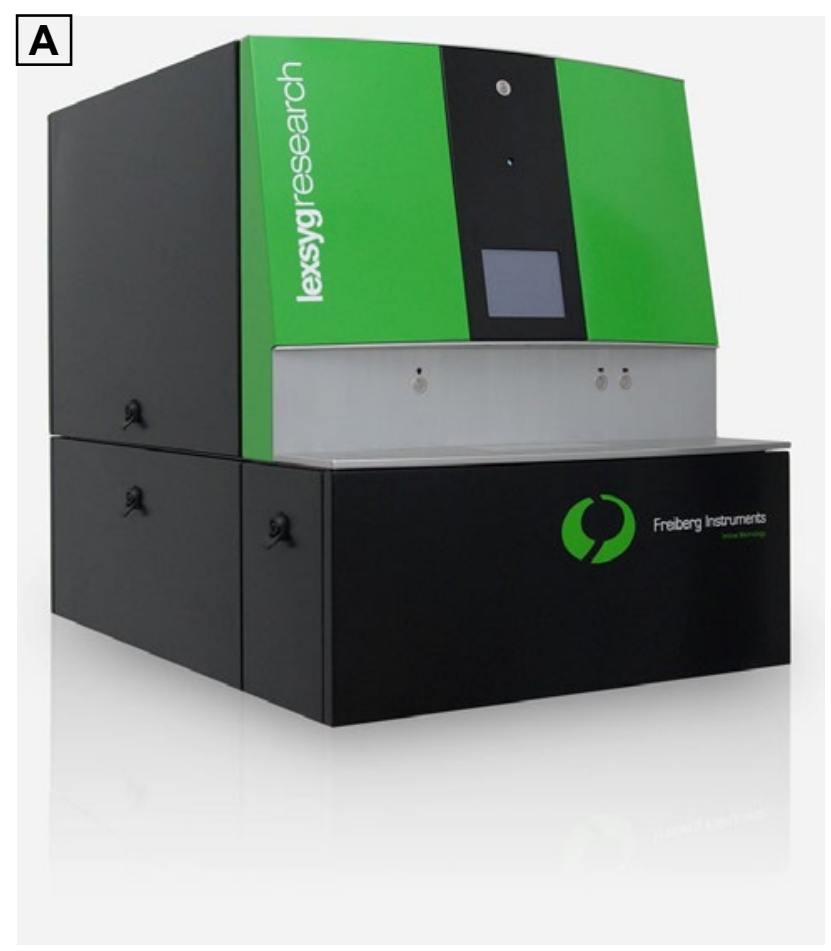

B

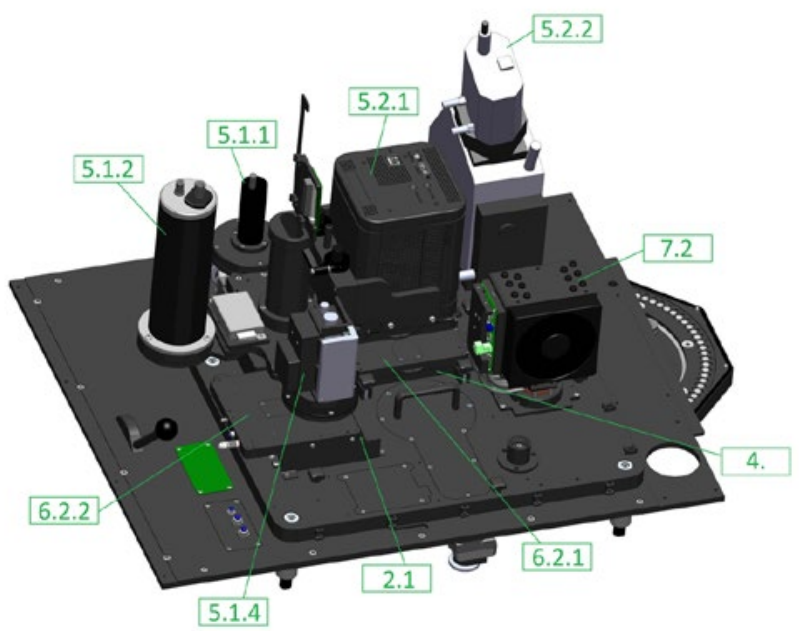

Fig. 2. The lexsyg luminescence system. A) Photograph of system, and B) three dimensional technical drawing of sample storage wheel (chamber) and closed measurement chamber with a selection of detection units, some of which mounted on a detector changer. Modules/units are numbered as follows: Filter Wheel on top of Beta-Irradiation unit (2.1); Optical Stimulation unit (4); UV-VIS PMT Module (5.1.1); Extended UV-VIS PMT Module (5.1.2); VIS-NIR PMT-Module (5.1.4); EMCCD luminescence Imaging module (5.2.1); CCD-Spectrometry unit (5.2.2); Imaging optics module for TL/OSL (6.2.1); Filter wheel (6.2.2); LED-based Solar Simulation unit (7.2).

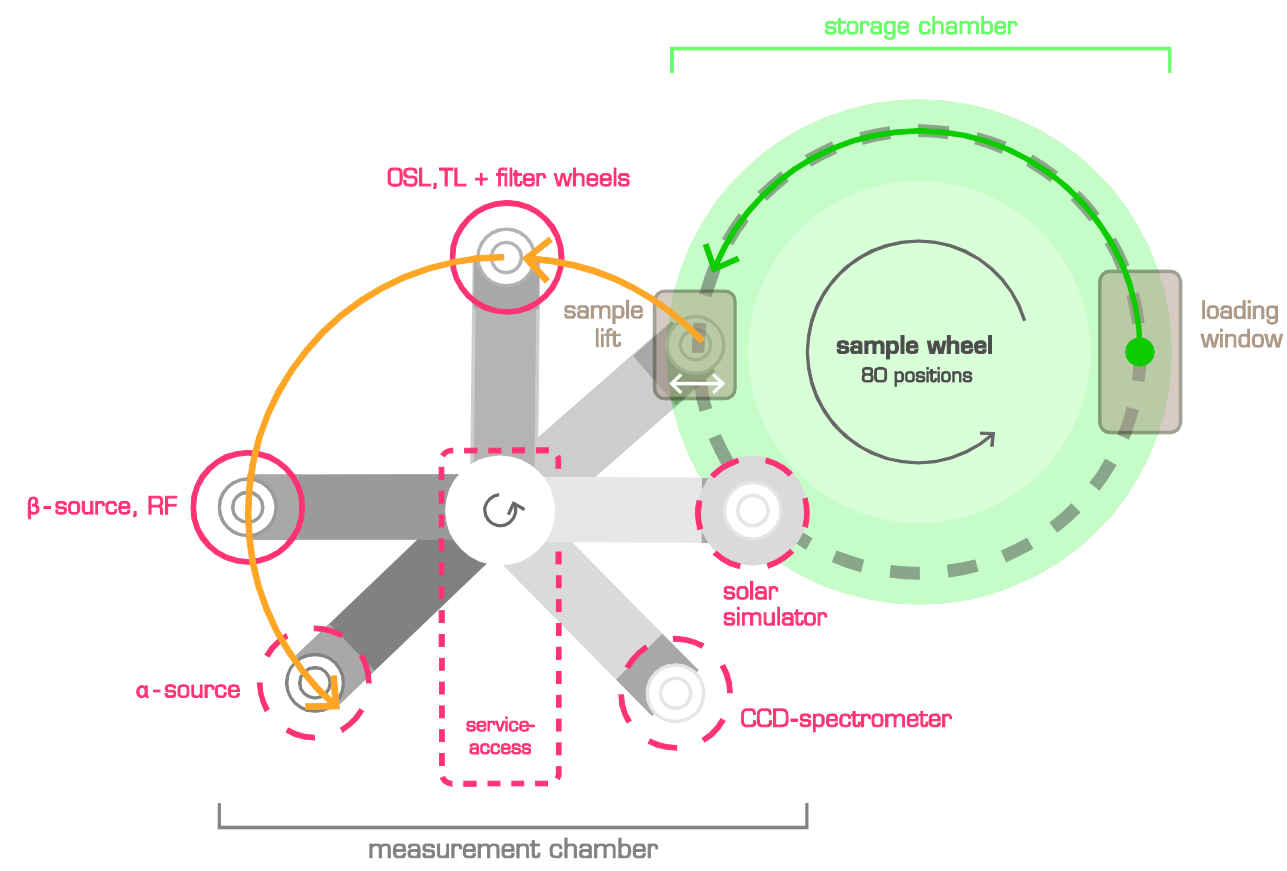

Fig. 3. Schematic drawing of the lexsyg system in which the sample aliquots are stored on a sample wheel separate from the measurement chamber, where an arm with the aliquot to be measured serves several positions for irradiation and/or stimulation. 
Rotational movements of the sample storage wheel, the detector changer and the measurement arm are realised by electrical stepping motors, while all other mechanical interactions are pneumatically driven. Typical sample cup thickness is $0.5 \mathrm{~mm}$ with a total allocation of maximum height (including cup and sample) of $1.5 \mathrm{~mm}$. The measurement chamber is separated from the storage wheel by a rubber seal, which is mechanically opened/closed during aliquot transfer. The aliquot is lifted up by a piston from the sample wheel, held in place by vacuum, picked up by a grabber and transferred to the heater plate on the sample arm in the measurement chamber (Fig. 4). Such sample changes are possible at nearly any time during a sequence.

The measurement chamber can be operated with air, $\mathrm{N}_{2}$ or He atmosphere, and be held slightly above atmospheric pressure. A pump provides flow within the closed system for cooling, which allows nitrogen consumption of approximately 2 1/aliquot for a standard SAR cycle and compressed air consumption of $31 /$ day, allowing low cost standard operation. Space for up to five positions is provided in the sealed chamber ( $\leq 1 \mathrm{mbar}$ ). These can hold up to two irradiation sources ( $\alpha$ and $\beta$ ) and/or are available for detection or bleaching modules, each of which can be fitted with a quartz window (Sico SQ1). For luminescence detection an automatic changer is available, which allows the software driven change of four different detection units, serving two measurement positions, without having to move the sample or requiring any user manipulation of the equipment (Fig. 5). The system is modular and in principle almost any additional detection/stimulation/irradiation module can be added at a later stage, including the detector wheel.

\section{Luminescence stimulation}

Luminescence can be stimulated by means of e.g. heating, exposure to light or ionising radiation for which the lexsyg system provides a wide range of excitation sources.

\section{Thermal stimulation}

For thermal luminescence stimulation (TL) a heating plate consisting of a metal plated dielectric heater was developed, which is capable of heating at rates of 0.1$20.0 \mathrm{~K} \mathrm{~s}^{-1}$ (@ $\mathrm{T}_{\max }=400^{\circ} \mathrm{C}$ ) and 0.1-10.0 K s${ }^{-1}$ (@) $\mathrm{T}_{\max }=700^{\circ} \mathrm{C}$ ). Temperature calibration is obtained for each individual heater by pyrometer measurements. By implementation of a closed loop gas flow system the cooling of the heater can be well controlled and the heating/cooling can be done at the specified rates, including non-linear heating/cooling functions, for an almost unlimited number of ramping, staging and cooling. TL or elevated temperature OSL/IRSL measurements can be performed up to $700^{\circ} \mathrm{C}$.

\section{Optical stimulation}

Optical stimulation in the lexsyg can be achieved by a variety of light excitation sources of different wavelengths. LEDs are available which have an emission bandwidth of 50 to $150 \mathrm{~nm}$, thus challenging the ability to block tail band light for any measurement window. This requires filtering, and thus reducing the emission bandwidth in order to obtain narrower excitation, at the loss of power delivered at the sample position. Rise and fall time turning LED light on and off is physically limited to around $4 \times 10^{-3} \mathrm{~s}$.

Alternatively laser diodes are available for some excitation bandwidths of 2-5 $\mathrm{nm}$ at higher optical dissipation power, which allows a physically well-defined carrier

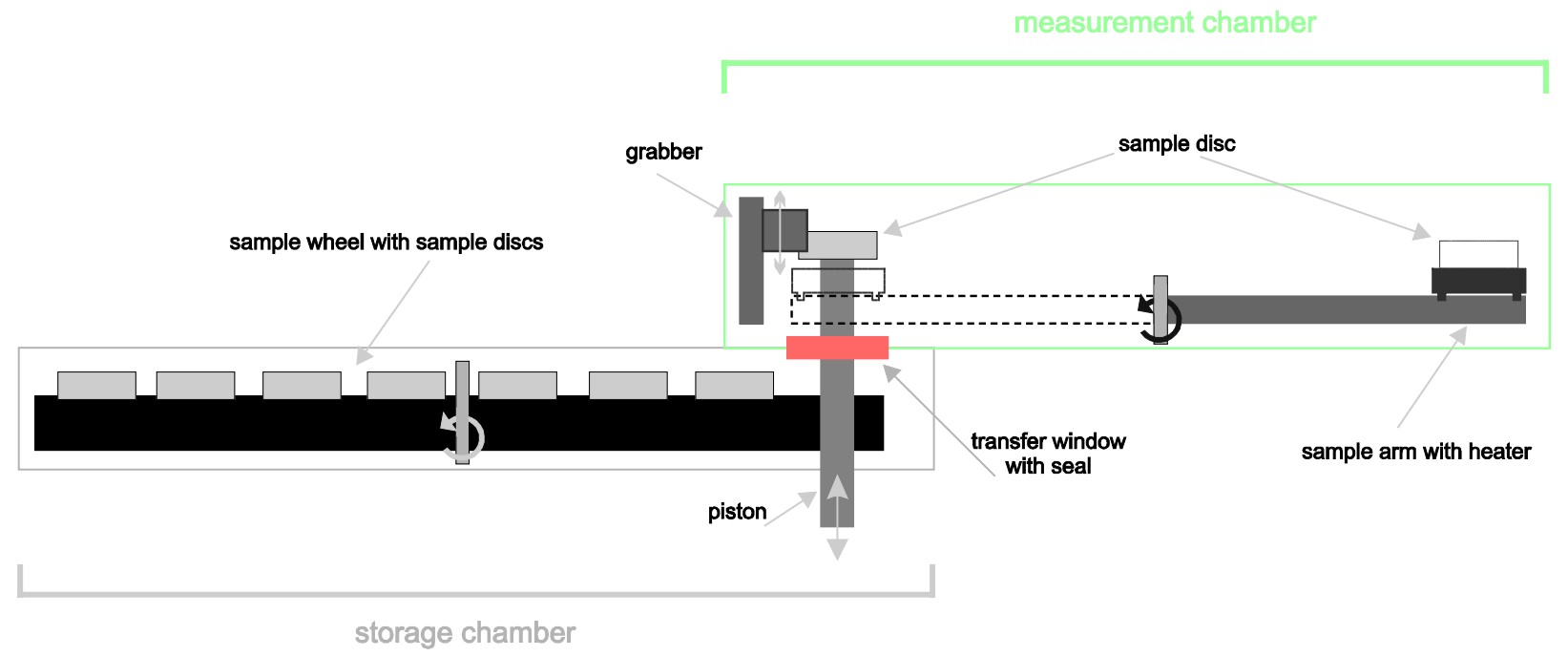

Fig. 4. Schematic section through the sample storage chamber with the sample wheel and the sample arm with heater plate in the measurement chamber, showing the lift and pick-and-place system used to transfer the sample. 


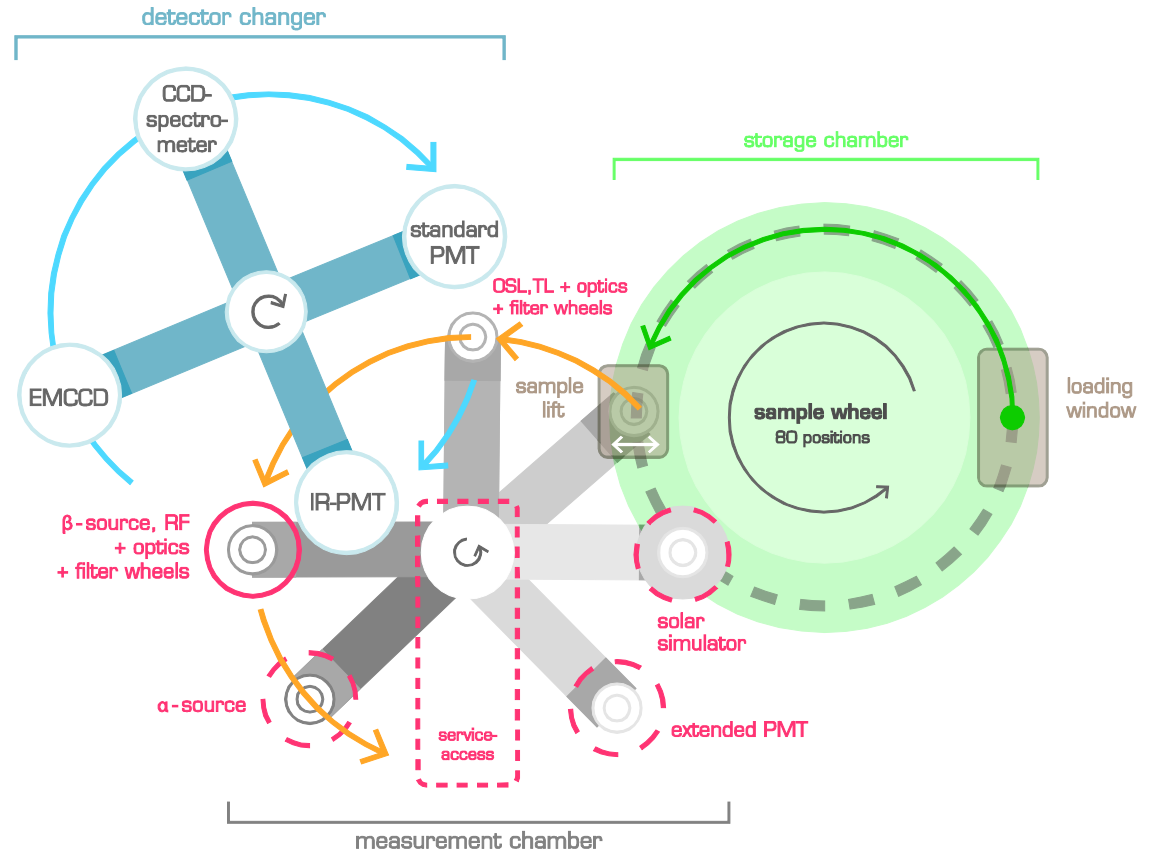

Fig. 5. Schematic drawing of the lexsyg system with detector changer.

excitation out of traps within the sample. Laser diode emissions are oriented within a certain numerical aperture and are focused in the lexsyg by suitable optics. Detection filter selection and defined blocking of the excitation light during detection is more effective, compared to LEDs, due to the small emission bandwidth. Fast rise time can be achieved $(<4 \mathrm{~ns})$ allowing fast pulsing as well as continuous wave $(\mathrm{CW})$ experiments with the same unit. For optical power control all optical excitation devices are actively monitored and currents are regulated to ensure stable optical emission over the years.

In a standard configuration an IR laser diode $(850 \pm 3 \mathrm{~nm})$ is mounted to homogeneously illuminate the sample area of $8 \mathrm{~mm}$ diameter with up to $400 \mathrm{~mW} \mathrm{~cm}$ via glass fibre optics (bundle of $1 \mathrm{~mm}$ diameter). Since the optical profile is Gaussian on such bundles all fibres are crossed for homogenisation and afterwards separated into eight fibres. Seven of those fibres are evenly arranged about $51^{\circ}$ apart at a certain angle and distance from the sample. Based on the emission profile, finite element optical simulation provides the optimal geometrical fibre position. The last fibre of $5 \mu \mathrm{m}$ diameter is coupled into a photodiode for active power control.

Similar setups are available for blue and green lasers as well, but a different configuration is needed for UV excitation due to defect generation within the glass fibre bundle, which is causing a degradation of the fibre within less than 1000 hours depending on laser power. Therefore direct sample illumination is chosen for a violet laser diode at $405 \pm 5 \mathrm{~nm}$ (filtered by Edmund Optics NT65072) which provides approximately $350 \mathrm{~mW} \mathrm{~cm}^{-2}$ at the sample position. Quartz stimulation is achieved by 5 green LEDs directly delivering approximately $60 \mathrm{~mW}$ $\mathrm{cm}^{-2}$ to the sample area, with excitation restricted by a $3 \mathrm{~mm}$ Schott OG515 and a Brightline HC530/43 filter to $525 \pm 25 \mathrm{~nm}$. Alternatively, 5 blue LEDs filtered by $3 \mathrm{~mm}$ Schott GG455 glass and a bandpass $458 \pm 10 \mathrm{~nm}$ filter (Edmund Optics 65-142) are delivering approximately $100 \mathrm{~mW} \mathrm{~cm}{ }^{-2}$. With the exception of green lasers, all diode lasers/LEDs can be operated independently in $\mathrm{CW}$ or modulated mode (linear/non-linear), with the possibility to couple an external light source as well. So far selectable pulse widths of $\geq 5 \mu$ s up to $\mathrm{CW}$ at rise and fall times of $<3 \mu \mathrm{s}$ have been demonstrated for LEDs. For laser diodes, up to two orders of magnitude faster rise and fall times with corresponding shorter pulse widths were achieved with technology transferred from similar applications in semiconductor research as in Berger et al. (2011). It is obvious that the time resolution of the transient measurement system must be able to account for these very short switching times of the light source.

\section{Light Irradiation Units}

A multi-wavelength power-LED-array based "solarsimulator" can deliver light of approximately $400 \mathrm{~mW}$ optical power (Table 1), which can be individually varied for six wavelength bands provided by violet-, blue-, green-, yellow-, red- and IR-LED-arrays. This allows the simulation of near natural sunlight or other light exposure conditions, like underwater, etc. Three more simple arrays for bleaching consist of either UV, RGB A (462, 523,590 and $625 \mathrm{~nm}$ peak wavelength) or IR LEDs 
(Table 1), which can only be varied in intensities of the whole unit.

\section{Ionising stimulation}

The Beta $\left({ }^{90} \mathrm{Sr}-{ }^{90} \mathrm{Y} ; 1.6 \mathrm{GBq}\right)$ irradiation unit was specially designed to provide a homogeneous irradiation field and to allow RL (in particular radiofluorescence, RF) measurements during beta irradiation because of its design as a ring source. Details especially on the homo-

Table 1. Optical power from LED bleaching units for the various wavelengths. Note that maximum intensity wavelengths are given, the actual wavelength range of the unfiltered LEDs is wider.

\begin{tabular}{ccc}
\hline $\begin{array}{c}\text { wavelength } \\
(\mathrm{nm})\end{array}$ & $\begin{array}{c}\text { 'solar simulator' } \sim \text { max. } \\
\text { power }(\mathrm{mW})\end{array}$ & $\begin{array}{c}\text { bleaching unit } \sim \text { max. } \\
\text { power }(\mathrm{mW})\end{array}$ \\
\hline 365 & 65 & 350 \\
$\mathbf{4 6 2}$ & 140 & 690 \\
$\mathbf{5 2 3}$ & 35 & 200 \\
$\mathbf{5 9 0}$ & 50 & 250 \\
625 & 85 & 440 \\
850 & 170 & 600 \\
\hline
\end{tabular}

geneity of the radiation field of about $\pm 2 \%$ at $8 \mathrm{~mm}$ diameter of the irradiation area and conditional parameters can be found in Richter et al. (2012). For non-RL application less homogeneous irradiation from a $\beta$-source of slightly higher activity $(<2 \mathrm{GBq})$ can be used, which is achieving approximately 0.13 Gy $\mathrm{s}^{-1}$. Additionally, $\alpha$-irradiation is possible with a ${ }^{241} \mathrm{Am}$ source $(30 \mathrm{MBq})$.

\section{Luminescence detection}

Spectral analysis (e.g. Huntley et al., 1988; Krbetschek et al., 1997; Rendell et al., 1993; Townsend, 1994) have shown the need for restricting the detection window in luminescence application (Fig. 1), which is commonly achieved by filters in the light path of detection. Up to two filter wheels can be mounted for each detection module with 6 positions each providing the possibility for changing the detection window between measurements within seconds. The lexsyg system can, in principle, be equipped with up to 6 luminescence detection units and also hold an automatic four detector changer, which can switch detectors during measurement sequences (Fig. 6).

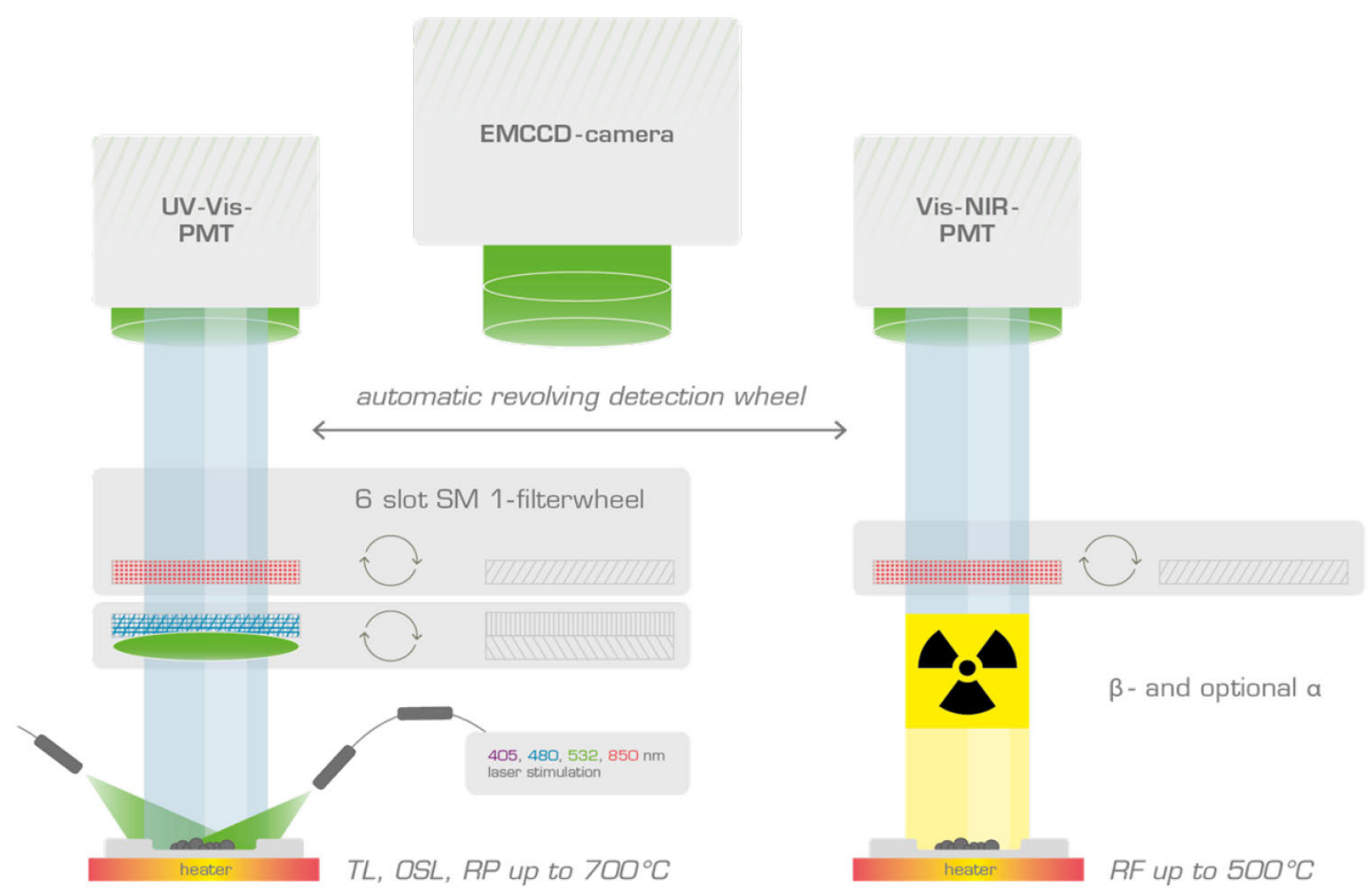

Fig. 6. Schematic setup of a lexsyg system with detector changer, which can switch up to four detection units automatically. 


\section{Photomultiplier}

Wavelength detection for standard bi-alkaline photomultiplier tubes from Hamamatsu (H7360-02) and EMI (9235QB) range 300-650 $\mathrm{nm}$ and 160-630, with quantum efficiencies of approximately $27 \%$ at $400 \mathrm{~nm}$ and $30 \%$ at 200 and $340 \mathrm{~nm}$, respectively (Fig. 1).

The beneficial use of an extended PMT for more efficient detection of luminescence emission at e.g. $620 \mathrm{~nm}$ for TL of quartz has been shown in a recent study (Richter et al., forthcoming). The Hamamatsu H7421-40 thermoelectric/air cooled GaAsP photocathode PMT is primarily sensitive between 300 and $720 \mathrm{~nm}$, with a peaksensitivity of $40 \%$ quantum efficiency at $580 \mathrm{~nm}$. The infrared radiofluorescence (IR-RF) of K-feldspar at $850 \mathrm{~nm}$ can be detected with the thermoelectric/air cooled $\left(-10^{\circ} \mathrm{C}\right) \mathrm{GaAs}$ photocathode PMT Hamamatsu H7421-50, which is mounted over the $\beta$-irradiation ring source (Richter et al., 2012). It provides a general sensitivity between 380 and $890 \mathrm{~nm}$, with a peak of $12 \%$ quantum efficiency at $800 \mathrm{~nm}$ (Fig. 1).

The dark count rates typically range between 50 and 300 counts s $\mathrm{s}^{-1}$ at $0^{\circ} \mathrm{C}$ for the different types of PMTs. For pulsed/time-resolved luminescence measurements special electronic for fast transients was developed, providing up to 4000 detection channels for user defined time widths $\geq 16$ ns.

\section{EMCCD}

Spatially resolved luminescence or single grain detection is possible with a Princeton ProEM:512B EMCCD camera (Figs. 7 and 8) either mounted in the changer or on a fixed position. Maximum resolution is $512 \times 512$

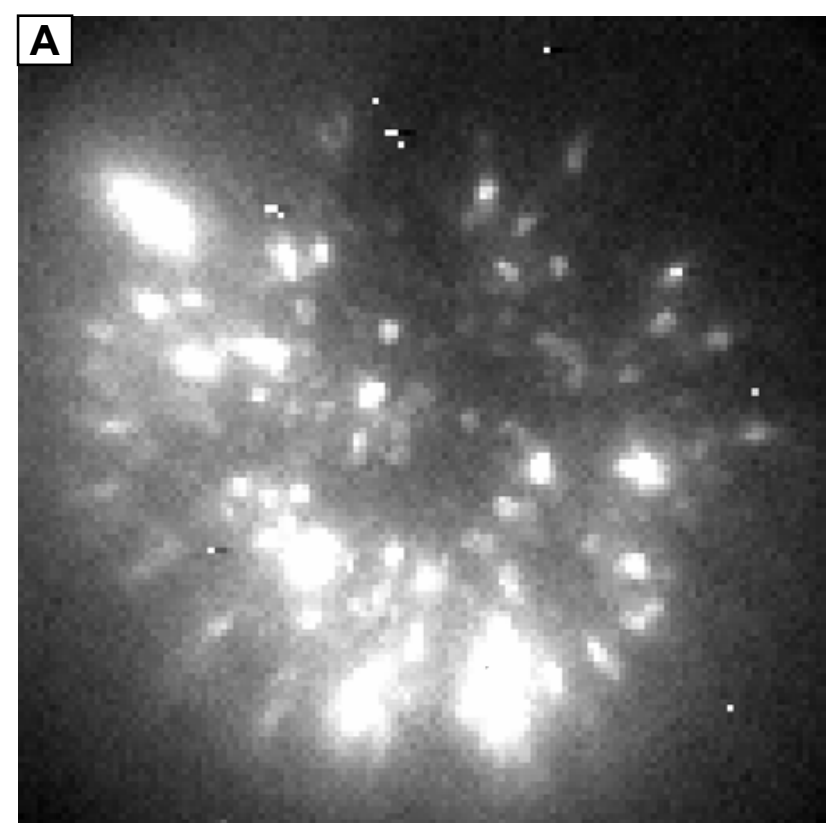

pixels at a chip size of $8.2 \times 8.2 \mathrm{~mm}$. With cooling to $-70^{\circ} \mathrm{C}$ and to be used in CCD mode for lowest noise possible at 16 bit resolution. Hardware binning is possible up to $8 \times 8$ pixels. The EMCCD based module provides high quantum efficiencies (up to 92\%) from UV to NIR (Fig. 1) and is aimed towards spatially resolved measurements of multiple grains or solid samples, but single-grain luminescence as well. Completely isotropic emission of the

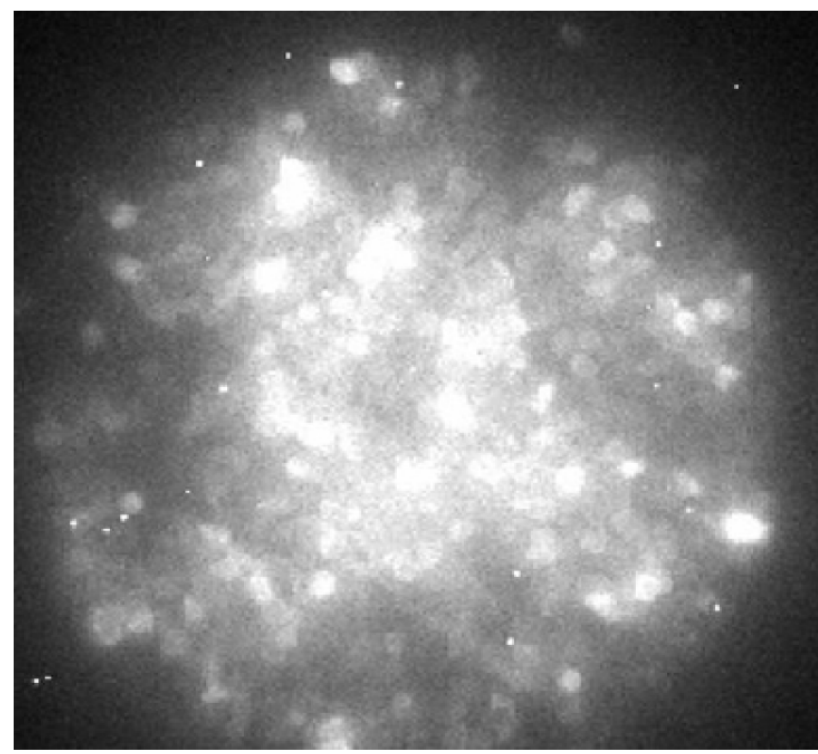

Fig. 7. Natural TL emission of Australian quartz (200-315 $\mu \mathrm{m})$ at $320^{\circ} \mathrm{C}(414 \mathrm{~nm} / 46 \mathrm{~nm}$ IF band-pass filter) detected with the lexsyg EMCCD (experimental conditions).

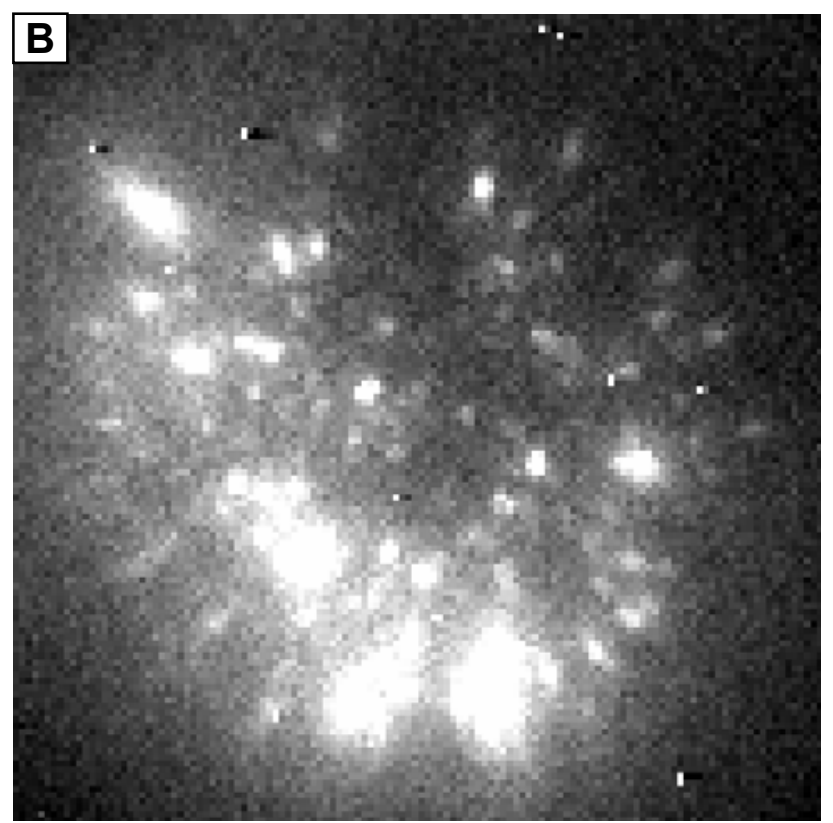

Fig. 8. EMCCD picture of the first $10 \mathrm{~s}$ OSL from quartz excited by $473 \mathrm{~nm}$ laser stimulation $\left(90-150 \mu \mathrm{m}\right.$, zeroed for $\left.300 \mathrm{~s} @ 125^{\circ} \mathrm{C}\right)$ and $\beta$-dose of A) 100 Gy and B) 10 Gy (untreated data). 
sample is a particular challenge. For the sake of sensitivity all optics must have a numeric aperture as large as possible with respect to resolution. Combination of large numerical aperture (up to 0.5 with UV-sensitive optics) and high resolution provides a particular challenge. Several optics were developed including corrections for certain spectral emissions. For bright emissions as RF, optics is optimised for IR emissions and resolution is in the focus of optics design. On the other hand for standard OSL emissions image resolution is less important in favour of highest quantum efficiency. Any excitation mode listed above can be used, including ionizing radiation for RF (Fig. 9). For data analysis the software AgesGalore (Greilich et al., 2006) can be employed.

\section{CCD-spectrometer}

Spectral luminescence analysis can be performed with a high-efficiency CCD based luminescence spectrometer (ANDOR Shamrock 163) with interchangeable gratings to provide TL (Fig. 10A) or OSL spectra (Fig. 10B) in the wavelength range of $200-1050 \mathrm{~nm}$. Here, the module is fixed because of its size and connected by a light guide, but can be operated with the detection changer in order to keep the flexibility of the system. The sensitivity of the TE-cooled (min. $-80^{\circ} \mathrm{C}$ ) CCD-spectrometer (iDus DU420A) depends on the grating with peak efficiencies of $60-90 \%$. Standard wavelength calibration is $350-$ $850 \mathrm{~nm}$, but gratings can be changed easily.

\section{CONCLUSIONS}

The lexsyg system provides a versatile tool for a wide variety of stimulation and detection configuration (Table 2) in luminescence fundamental research as well as dating application. It is capable of the excitation of luminescence by thermal as well as violet, blue, green, IR and ionizing stimulation. The power of the equipment is in the versatility of available detection modules. A variety of photomultiplier tubes for different applications (e.g. orange-red or IR-RF detection) can be employed and an EMCCD camera allows spatial luminescence analysis, while a CCD-spectrometer is provided for fundamental studies of luminescence emissions. Detection wavelength bands can be automatically changed with up to two 2 filter wheels holding 6 slots each at almost any detection position. Even detectors can be automatically changed. Such changes are possible within nearly all measurement sequences without user interference. No manipulation of

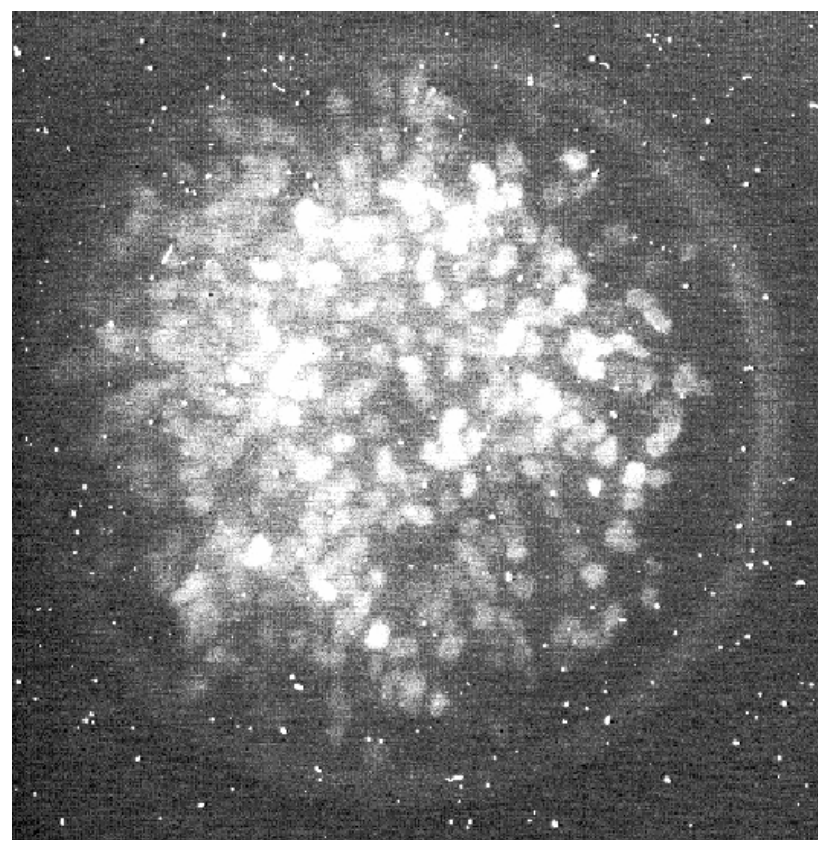

Fig. 9. EMCCD picture of $0-20 \mathrm{~s}$ of IR-RF from a K-feldspar with detection window restricted by a Chroma ET 850/30 filter.
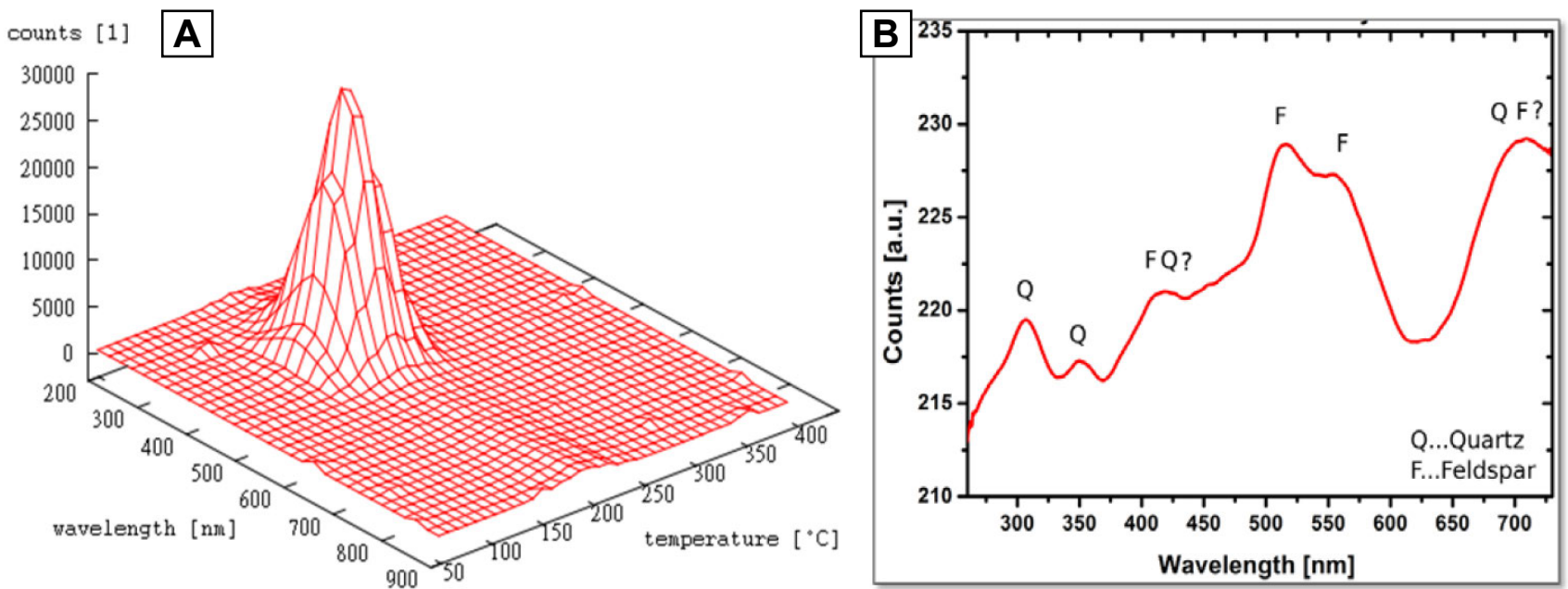

Fig. 10. Smoothed thermoluminescence emission spectra of $A) \alpha-A l_{2} \mathrm{O}_{3}: \mathrm{C}(50 \mathrm{~Gy})$ and $B$ ) a polymineral sediment sample ( $\mathrm{F}=$ feldspar and $Q=$ quartz emission) measured with a Shamrock 163 spectrometer (Heinz, 2011). 
Table 2. Summary of excitation and stimulation modules available for the lexsyg system.

\begin{tabular}{|c|c|c|}
\hline stimulation & wavelength band (nm) & power $\left(\mathrm{mW} \mathrm{cm}^{-2}\right)$ \\
\hline green LEDs & 525 & 60 \\
\hline blue laser diode & 465 & 100 \\
\hline IR -laser diode & 850 & 200 \\
\hline violet laser diode & 405 & 350 \\
\hline detection & wavelength band (nm) & quantum efficiency \\
\hline $\begin{array}{l}\text { Hamamatsu PMT } \\
\text { (bialkaline) }\end{array}$ & $300-650$ & @ 400 nm: 27\% \\
\hline $\begin{array}{l}\text { EMI PMT } \\
\text { (bialkaline) }\end{array}$ & $160-630$ & $\begin{array}{l}\text { @ } 200 \text { nm: } 30 \% \\
\text { @ } 340 \text { nm: } 30 \%\end{array}$ \\
\hline $\begin{array}{c}\text { Hamamatsu PMT } \\
(\text { GaAsP })\end{array}$ & $300-720$ & @ 580 nm: 40\% \\
\hline $\begin{array}{c}\text { Hamamatsu PMT } \\
(\mathrm{GaAs})\end{array}$ & $380-890$ & @ 800 nm: 12\% \\
\hline CCD & $200-1050$ & $\begin{array}{l}\text { @ } 340 \text { nm: } 30 \% \\
\text { @ } 770 \text { nm: } 58 \%\end{array}$ \\
\hline EMCCD & $200-1100$ & $\begin{array}{l}\text { @ } 350 \text { nm: } 35 \% \\
\text { @ } 600 \text { nm: } 96 \%\end{array}$ \\
\hline
\end{tabular}

the equipment is required because all changes are software controlled. This will allow more diverse ways to perform luminescence stimulation and detection, which was not possible previously. By providing such a versatile tool many avenues for new developments in luminescence research are expected to be opened.

\section{ACKNOWLEDGEMENTS}

We would like to thank R. DeWitt and U. Rieser for providing sample material and help with measurements, and the entire engineering and scientific team at Freiberg Instruments for making this development possible.

\section{REFERENCES}

Aitken MJ, 1985. Thermoluminescence Dating. London, Academic Press: 359pp.

Aitken MJ, 1998. An Introduction to Optical Dating. The Dating of Quaternary Sediments by the Use of Photon-stimulated Luminescence. Oxford University Press, Oxford: 280pp.
Berger B, Schüler N, Anger S, Gründig-Wendrock B, Niklas JR and Dornich K, 2011. Contactless electrical defect characterization in semiconductors by microwave detected photo induced current transient spectroscopy (MD-PICTS) and microwave detected photoconductivity (MDP). Physica Status Solidi (a) 208(4): 769-776, DOI 10.1002/pssa.201083994.

Bortolot VJ, 2000. A new modular high capacity OSL reader system. Radiation Measurements 32(5-6): 751-757, DOI 10.1016/S13504487(00)00038-X.

Bortolot VJ and Carriveau GW, 1982. An integrated TL measurement and computer system. PACT, Revue du groupe européen d'études pour les techniques physiques, chimiques et mathématiques appliquées à l'archéologie 6: 272-281.

Bøtter-Jensen L and Bundgaard J, 1978. An automated reader for thermoluminescent dating. PACT, Revue du groupe européen d'études pour les techniques physiques, chimiques et mathématiques appliquées à l'archéologie 2: 48-57.

Bøtter-Jensen L, McKeever SWS and Wintle AG, 2003. Optically Stimulated Luminescence Dosimetry. Amsterdam, Elsevier, 374pp.

Greilich S, Harney HL, Woda C and Wagner GA, 2006. AgesGalore - A software program for evaluating spatially resolved luminescence data. Radiation Measurements 41(6): 726-735, DOI 10.1016/j.radmeas.2005.12.007.

Heinz D, 2011. Optimierung einer Spektroskopieeinheit zur automatisierten Lumineszenzmessung. Minor Thesis, Faculty of Materials Science and Technology, Technical University Bergakademie Freiberg.

Huntley DJ, Godfrey-Smith DI, Thewalt MLW and Berger GW, 1988. Thermoluminescence spectra of some mineral samples relevant to thermoluminescence dating. Journal of Luminescence 39(3): 123136, DOI 10.1016/0022-2313(88)90067-1.

Krbetschek MR, Götze J, Dietrich A and Trautmann T, 1997. Spectral information from minerals relevant for luminescence dating. Radiation Measurements 27(5-6): 695-748, DOI 10.1016/S13504487(97)00223-0.

Lapp T, Jain M, Thomsen KJ, Murray AS and Buylaert J-P, 2012. New luminescence measurement facilities in retrospective dosimetry. Radiation Measurements 47(9): 803-808, DOI 10.1016/j.radmeas.2012.02.006.

McKeever SWS, 1985. Thermoluminescence of solids. Cambridge, Cambridge University Press, 392.

Mejdahl V, 1982. An automated procedure for the thermoluminescence dating of pottery and burnt stones. PACT, Revue du groupe européen d'études pour les techniques physiques, chimiques et mathématiques appliquées à l'archéologie 7, 83-96.

Rendell HM, Khanlary MR, Townsend PD, Calderón T and Luff BJ, 1993. Thermoluminescence spectra of minerals. Mineralogical Magazine 57: 217-222.

Richter D, Pintaske R, K.Dornich and Krbetschek M, 2012. A novel beta source design for uniform irradiation in dosimetric applications. Ancient TL 30(2): 57-63.

Townsend PD, 1994. Analysis of TL emission spectra. Radiation Measurements 23(2-3): 341-348, DOI 10.1016/1350-4487(94)90062-0.

Yukihara EG and McKeever SWS, 2011. Optically stimulated luminescence: Fundamentals and applications. Oxford, Wiley-Blackwell, $388 \mathrm{pp}$. 